\title{
Long-Lifetime, Low-Contamination Metal Beam Dumps for NIF Spatial Filters
}

\author{
M. A. Norton \\ C. D. Boley \\ J. E. Murray \\ K. Sinz \\ K. Neeb
}

This paper was prepared for submittal to the

Third Annual International Conference on

Solid State Lasers for Application (SSLA)

to Inertial Confinement Fusion (ICF)

Monterey, California

June 7-12, 1998

October 30, 1998

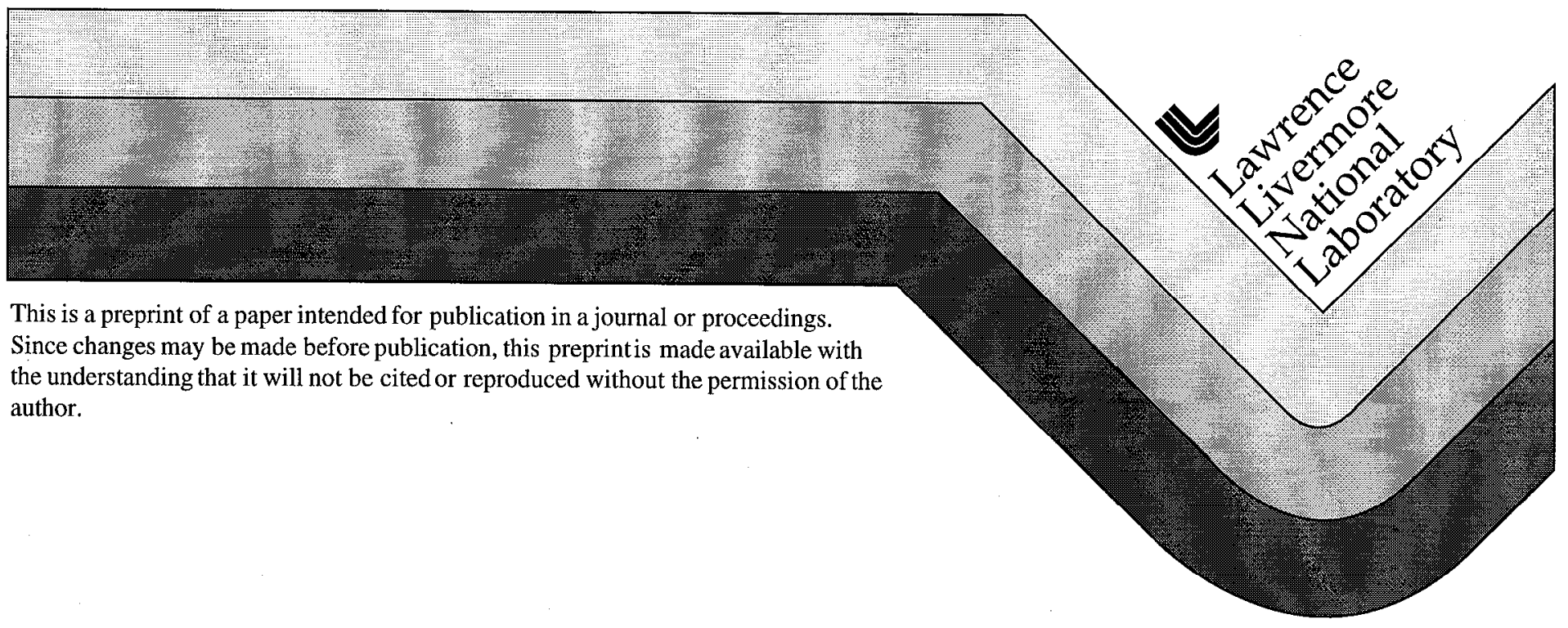




\section{DISCLAIMER}

This document was prepared as an account of work sponsored by an agency of the United States Government. Neither the United States Government nor the University of California nor any of their employees, makes any warranty, express or implied, or assumes any legal liability or responsibility for the accuracy, completeness, or usefulness of any information, apparatus, product, or process disclosed, or represents that its use would not infringe privately owned rights. Reference herein to any specific commercial product, process, or service by trade name, trademark, manufacturer, or otherwise, does not necessarily constitute or imply its endorsement, recommendation, or favoring by the United States Government or the University of California. The views and opinions of authors expressed herein do not necessarily state or reflect those of the United States Government or the University of California, and shall not be used for advertising or product endorsement purposes. 
Long-lifetime, low-contamination metal beam dumps

for NIF spatial filters

\author{
Mary A. Norton, Charles D. Boley, James E. Murray, Kurt Sinz, and Kurt Neeb
}

University of California, Lawrence Livermore National Laboratory

P.O. Box 808, L-479, Livermore, CA 94550

\begin{abstract}
The management of back reflections and leakage through the Pockels cell and polarizer switch in the National Ignition Facility (NIF) beamlines requires robust beam dumps that can operate unattended for thousands of shots within the confines of the vacuum spatial filters. Off-line experiments and modeling have been used to investigate the use of metal for these beam dumps. We conclude that a stainless steel beam dump will have sufficient lifetime and will safely absorb fluences in the required range, from $1 \mathrm{~J} / \mathrm{cm}^{2}$ to $4 \mathrm{~kJ} / \mathrm{cm}^{2}$, at $1 \mu \mathrm{m}$.
\end{abstract}

\title{
1. INTRODUCTION
}

The NIF laser architecture must accommodate efficient trapping of unwanted beams that have their origin in Pockels cell leakage and target and/or pinhole backreflections. Since the NIF architecture uses angle multiplexing to make multiple passes through the final amplifiers, certain locations are appropriate for separation of unwanted beams from the main laser beam. These are within the vacuum spatial filters at and near the pinhole planes. Consequently, the fluences which the dumps must absorb are quite large, beyond the damage threshold of optical materials. We have used off-line tests, modeling results, and NOVA shots to develop a robust beam dump concept for NIF, capable of operation at more than $4 \mathrm{~kJ} / \mathrm{cm}^{2}$ and having a lifetime greater than 1000 shots.

The origin of the unwanted beams and the location of the required beam dumps can be seen in the schematic of the NIF architecture shown in Figure 1. The main beam is 


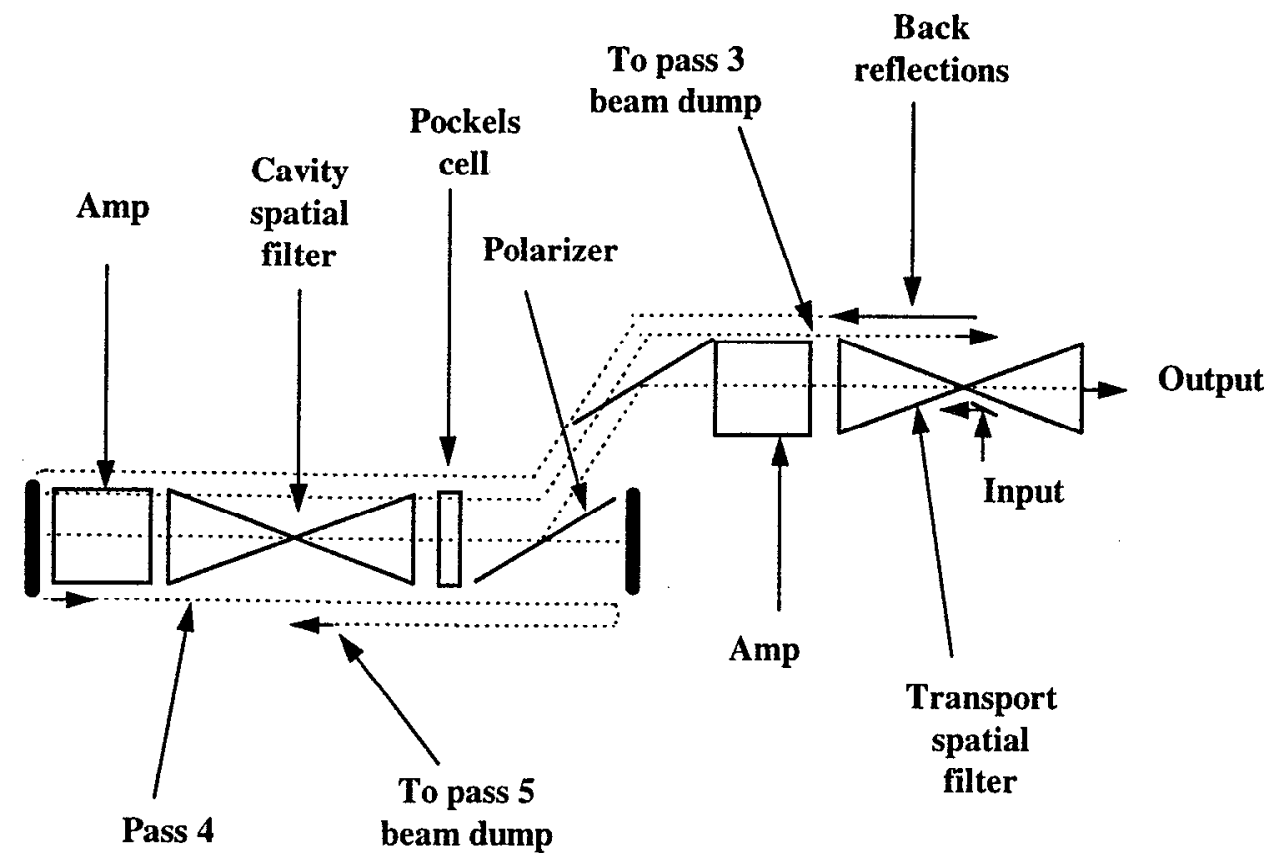

Figure 1. Schematic of the NIF laser architecture.

injected near the pinhole plane of the transport spatial filter (TSF). It then passes through the booster amplifiers into the 4-pass cavity by reflection from a polarizer. The Pockels cell is activated to keep the beam in the cavity for four passes through the cavity amplifiers before it is deactivated to return the main beam to the booster amplifiers. The combined performance specifications of the polarizer and Pockels cell allow $0.01 \%$ leakage into the cavity after the fourth pass. The path of the residual beam in the cavity, which makes a fifth pass after the main beam is sent out, is shown in Figure 1, where it is drawn below the path of the main beam. Since each pass is separated from the adjacent pass by a small angle, on the order of $1 \mathrm{mrad}$, the residual beam is directed toward a new location in the cavity spatial filter pinhole plane, and it has its own pinhole. The required beam dump will be located just beyond this pinhole.

The portion of the main beam which is back-reflected at the target, along with that which is back-reflected from the output pinhole of the TSF, can return along the outgoing path in the outgoing polarization state. After completing a round trip through the booster amplifiers and the cavity amplifiers, such a beam will arrive at a displaced position in the pinhole plane of the TSF. We designate this location as the pass 3 pinhole. This path is 
shown in Figure 1, displaced above the main beamline. The back-reflection beam dump will be located just beyond this pinhole.

In order to specify the performance requirements of these beam dumps, we must estimate the energy contained in the unwanted beams and the spatial volumes available for the dumps. To ensure that the beam dump does not obstruct other beam lines, we have imposed an exclusion zone of minimum width $5 \mathrm{~mm}$ between adjacent beams, as indicated in Figure 2. Thus the dumps should be located as far from the pinhole plane as possible in keeping with this constraint, to minimize the fluence of the leakage or back-reflected beams on the dump.

In the case of the leakage dump for pass 5, the expected energy is determined from the performance specifications of the Pockels cell and polarizer. These permit a maximum leakage of $.01 \%$ into pass 5 . The worst-case scenario would result in $150 \mathrm{~J}$ leaking into pass 5 . Although this full energy is not expected on every shot, some leakage energy will inevitably arrive on every laser shot.

In the case of the back-reflection dump in the TSF, it is necessary to make an estimate of the possible energy that may be reflected from the output spatial filter pinhole or the target. Calculations indicate that a $0.1 \%$ back-reflection will produce $2.5 \mathrm{~kJ}$ in this location. Since back-reflections from pinholes will occur only in the case of a major misalignment, and target reflections at $1 \omega$ are judged to be unlikely, this beam dump is not expected to receive appreciable energy on every shot.

The geometry near the pinhole plane of the vacuum spatial filters is indicated schematically in Figure 2, where $d$ is the spacing between adjacent pinholes. In principle,

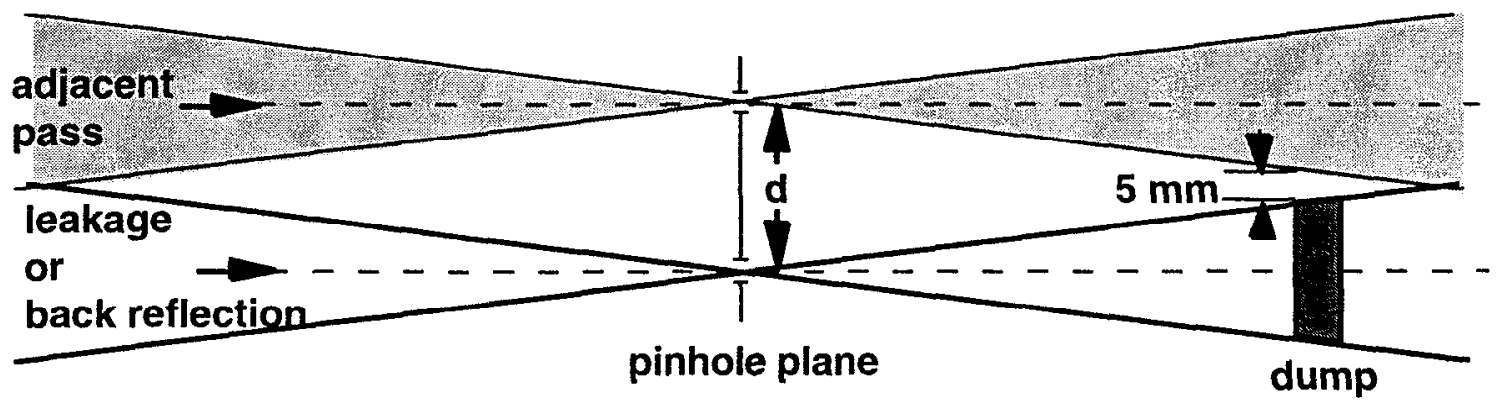

Figure 2. Schematic of spatial filter pinhole area. 
the width of the beam dumps could be as large as $\mathrm{d}-5 \mathrm{~mm}$ and still be consistent with the exclusion criterion. However, space conflicts within the vacuum spatial filter do not allow dumps to be located in the corresponding positions. The fluences in the permitted beam dump planes fall in the range $1-4 \mathrm{~kJ} / \mathrm{cm}^{2}$, as shown in Table 1 .

\begin{tabular}{|c|c|c|c|c|}
\hline Position & $\begin{array}{c}\mathbf{d} \\
(\mathbf{c m})\end{array}$ & $\begin{array}{c}\text { Energy } \\
(\mathbf{J})\end{array}$ & $\begin{array}{c}\text { Area } \\
\left(\mathbf{c m}^{2}\right)\end{array}$ & $\begin{array}{c}\text { Fluence } \\
\left(\mathbf{J} / \mathbf{c m}^{2}\right)\end{array}$ \\
\hline TSF & 3.5 & 2500 & 0.625 & 4000 \\
\hline CSF & 1.4 & 150 & 0.125 & 1200 \\
\hline
\end{tabular}

Table 1

These fluence estimates, along with the requirement of survivability without maintenance for at least 1000 shots, provide the design requirements for the beam dumps.

Since there are no known optical materials that have optical damage thresholds in the predicted range of fluences, we have chosen to use metals. Our approach has been to rely primarily on off-line small-scale tests, along with modeling, to develop a feasible design. The experiments were also designed to measure potential contamination of components in the spatial filters.

\section{EXPERIMENTS AND MODELING}

We used off-line tests to determine the amount of material ablated, and the spatial distribution, from a sample beam dump. These tests utilized a solid state laser system ${ }^{1}$ of wavelength $1.053 \mu \mathrm{m}$, operating at $1 \mathrm{~Hz}$ with $20 \mathrm{~J}$ per pulse. The pulse width was $16 \mathrm{~ns}$ FWHM, and the beam was 1.2 times diffraction-limited. The beam area on target ranged from $1 \mathrm{~mm} \times 1 \mathrm{~mm}$ to $4 \mathrm{~mm} \times 4 \mathrm{~mm}$, with the area selected to achieve the desired fluence. Samples were mounted in a vacuum system operating in the range of $10^{-5}$ torr. The angle of incidence was $45^{\circ}$ in some cases and $60^{\circ}$ in others. At the end of a run, the material lost by the sample was determined by weighing, and the result was checked with a microscopic measurement of the ablated volume. An arrangement of glass microscope slides surrounding the beam dump was used to collect ablation products throughout a run, which typically consisted of 1000 shots. The slides were masked to produce a sharp step between the deposited material and the masked, clear slide. A stylus profilometer was used to 
determine the thickness of the deposited layer as a function of angle from the surface normal.

The selection of potential beam dump materials was guided by the results of calculations, described below, which indicated that stainless steel, tantalum, and tungsten would ablate less than $1 \mu \mathrm{m}$ for fluences below $1 \mathrm{~kJ} / \mathrm{cm}^{2}$. Also initially considered was aluminum, but it was predicted to yield excessive removal of material.

The ablation of electropolished stainless steel, type 304, was measured over four orders of magnitude, from $1 \mathrm{~J} / \mathrm{cm}^{2}$ to $10 \mathrm{~kJ} / \mathrm{cm}^{2}$. All of the data for stainless steel, along with limited data obtained for tantalum and tungsten, are shown in Figure 3. The ablation depth in microns per shot versus laser fluence is plotted. The indicated fluence is normal to the beam propagation direction. The experimental points are plotted individually, and the model results are shown as lines.

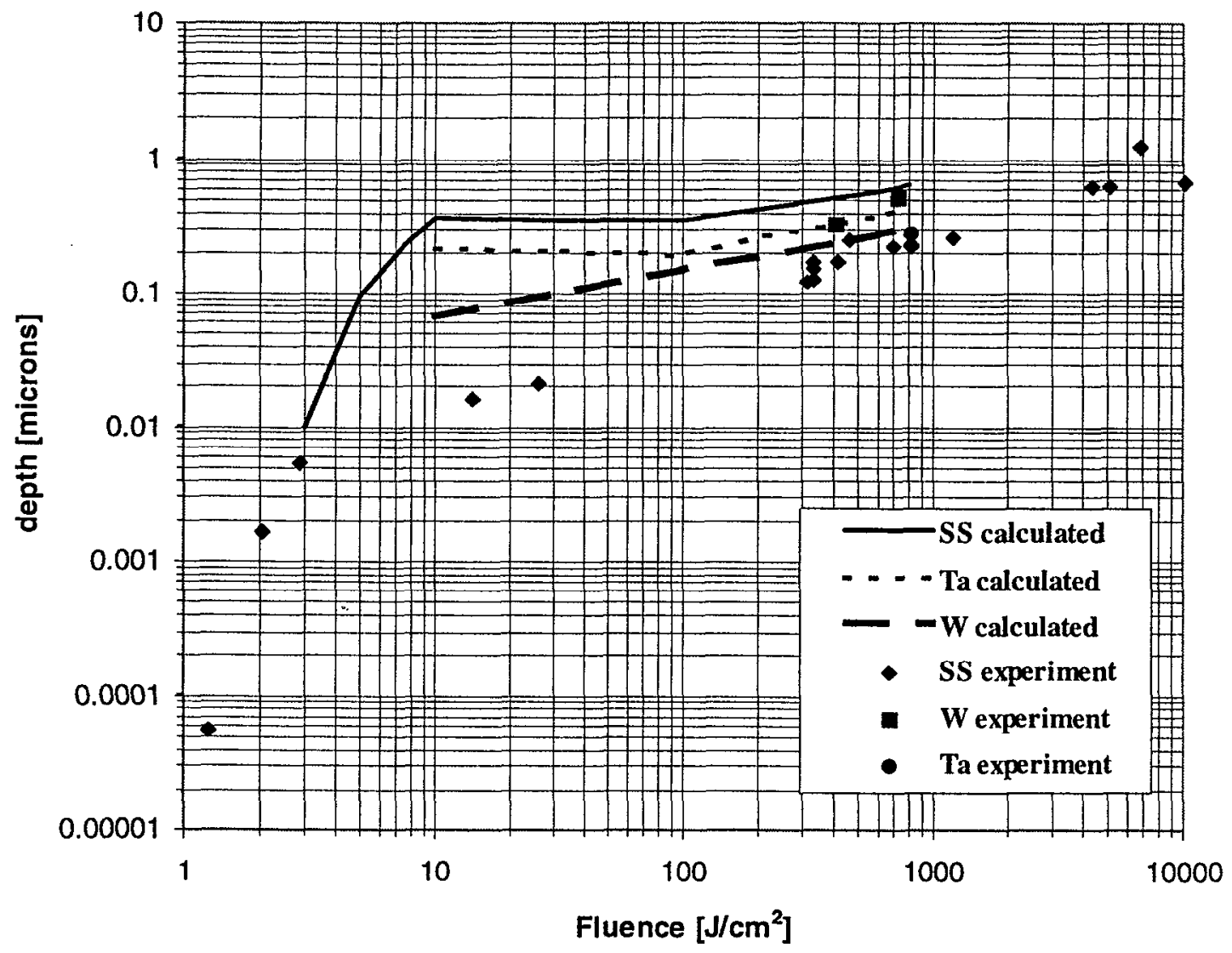

Figure 3. Laser ablation per shot versus laser fluence. 
The following are general observations concerning the data. Two angles of incidence are included in the stainless steel data, $45^{\circ}$ and $60^{\circ}$, but no difference in depth per shot was measured. To ensure that the measured depths were not influenced by thermal memory, we varied the shot rate from $0.1 \mathrm{~Hz}$ to $1 \mathrm{~Hz}$ and found no change. To determine if the recondensed material at the edge of the hole influenced the manner in which the material ablated from the surface, runs with 10 shots, 100 shots, 500 shots, 1000 shots were included in the above data. No difference in removal depth was found. The experimental point at approximately $7000 \mathrm{~J} / \mathrm{cm}^{2}$ shows an anomalously large removal depth, but it was obtained from the sole sample that ablated all the way through. Finally, the point at $10^{4} \mathrm{~J} / \mathrm{cm}^{2}$ was a single shot obtained from the NOVA laser. Altogether, the results indicate that less than $1 \mu \mathrm{m}$ of material is removed per shot over the entire range tested. Essentially no material is removed below $2 \mathrm{~J} / \mathrm{cm}^{2}$.

We modeled this experiment with a 1-D code ${ }^{2}$ which describes the initial illumination via the laser pulse, followed by the formation of a melt layer, ablation, and expansion of vaporized material. The model treats heat transport into the material, gas dynamics in the vapor, and light propagation through the vapor, while keeping track of the location of the melting surface and ablation surface. Vaporization is modeled by imposing the hydrodynamic jump conditions along the liquid-vapor coexistence curve. In the vapor, a Saha equation of state accounts for the pressure and internal energy resulting from ionization. Absorption in the vapor is due to photoionization (at relatively low temperatures) and inverse bremsstrahlung (at temperatures above a few $\mathrm{eV}$ ). In the simulations reported here, the critical electron density of about $1 \times 10^{21} \mathrm{~cm}^{-3}$ was never reached. After the passage of the light pulse, typical vapor temperatures ranged from $0.3 \mathrm{eV}\left(\right.$ at $3 \mathrm{~J} / \mathrm{cm}^{2}$ ) to $10 \mathrm{eV}$ (at $800 \mathrm{~J} / \mathrm{cm}^{2}$ ).

Referring to the stainless steel predictions in Figure 3, we see that the behavior falls into three regimes. For fluences up to about $10 \mathrm{~J} / \mathrm{cm}^{2}$, the ablation increases rapidly with fluence. Between $10 \mathrm{~J} / \mathrm{cm}^{2}$ and $100 \mathrm{~J} / \mathrm{cm}^{2}$, the ablation remains nearly constant. Thereafter it slowly increases. In the first regime, light arrives at the surface practically unimpeded by the vapor, and ablation is quite efficient. Absorption depends on intensity 
rather than fluence itself, and appreciable absorption typically begins for intensities, normal to the surface, above $10^{8} \mathrm{~W} / \mathrm{cm}^{2}$. In this experiment, this corresponds to a fluence of about $10 \mathrm{~J} / \mathrm{cm}^{2}$, explaining the first knee in the curve. Between this point and $100 \mathrm{~J} / \mathrm{cm}^{2}$, an increased fluence is predicted only to deposit more energy in the vapor, with a fixed amount striking the surface. At higher fluences, though, the vapor becomes sufficiently ionized for electron thermal conduction to carry appreciable energy to the surface. This is the mechanism responsible for the final rise in the curve.

Experiment shows a sharp rise for low fluences, followed by a slow but steady increase. We interpret this as evidence of the first and third regimes, without a pronounced transition regime of constant ablation. In addition, calculated ablation depths exceed experiment by a factor ranging from 1.5 (at the lowest calculated point), to 20 (near 10 $\mathrm{J} / \mathrm{cm}^{2}$ ), to 3 (at the highest fluence). This disagreement is hypothesized to be caused by plasma effects which are still under study. As regards the material dependence, the model predicts that heavy metals such as tantalum and tungsten should ablate less than stainless steel. This behavior is consistent with the higher melting temperature and vaporization temperature, at a given pressure, of heavy metals. Experiment, however, indicates the opposite dependence. The reason is also under study.

In addition to ablation, another consideration in the long-term survivability of the bean dump is the possibility of catastrophic failure from laser-induced shock damage. This issue was also addressed with calculations and experiments. For a single shot at $10 \mathrm{~kJ} / \mathrm{cm}^{2}$, calculations predicted that a wall thickness of $2 \mathrm{~mm}$ would be sufficient to reduce the shock wave magnitude from its initial value, which could be large enough to exceed the yield strength of the material, to the $5 \mathrm{kbar}$ range, which is within the elastic wave regime of stainless steel. The results from the calculations were benchmarked with full-scale shots on the NOVA laser at 3 and $10 \mathrm{~kJ} / \mathrm{cm}^{2}$. For the $1.5 \mathrm{~mm}$ thick samples used in these shots, there was no indication of material rupture on the back surface, in reasonable agreement with the calculations. For comparison, a single shot at $0.527 \mu \mathrm{m}$ at $14 \mathrm{~kJ} / \mathrm{cm}^{2}$ was found to leave a laser beam imprint on the back surface of a $1.5 \mathrm{~mm}$ thick stainless steel sample.

Another requirement of the beam dump is that it must not contaminate optical surfaces in the vacuum spatial filter assembly. We assume that all internal optics are 
baffled whenever possible to eliminate any direct line of sight to the beam dump. This leaves only line-of-sight contamination of the spatial filter lenses as a concern. To investigate this aspect of the problem, we measured the ablation product angular distribution to estimate the extent of contamination. By measuring the thickness of the deposited material on the glass slides, we obtained the angular distribution of the ablated material in the equatorial plane about the surface normal. The deposits were observed to be symmetric in the perpendicular plane. The ablated material thickness was measured with a stylus profilometer with a resolution of about $10 \mathrm{~nm}$. Consequently, a typical shot series used 1000 shots to accumulate sufficient material for measurement. All measured angular distributions are strongly peaked about the surface normal and typically offset in the forward direction by $5^{\circ}$ to $15^{\circ}$. The measured distribution at $4.4 \mathrm{~kJ} / \mathrm{cm}^{2}$ can be seen in Figure 4. Here the thickness of the deposit caused by a single shot, normalized to a radius of $1 \mathrm{~cm}$, is plotted versus angle from the beam dump normal. The beam was incident at $+60^{\circ}$. The solid line shows a fit to the data with a $\cos ^{8} \alpha$ distribution.

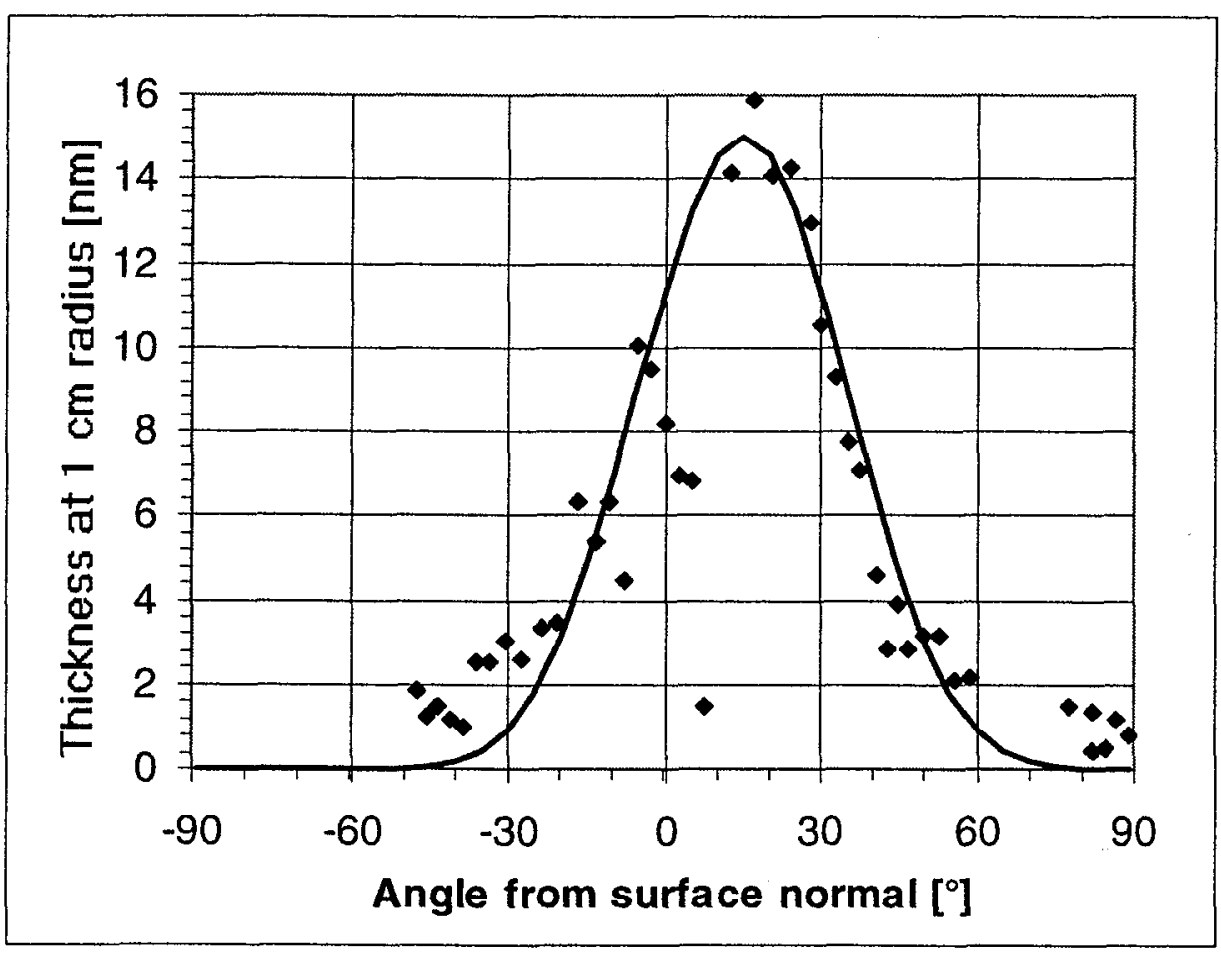

Figure 4 Ablation product distribution at $4.4 \mathrm{~kJ} / \mathrm{cm}^{2}$

Examination of the deposits on the slides with an optical microscope showed no evidence that particulates were launched from the surfacc. The material deposited on the 
slides was mirror-like and adhered so well that it could not be removed by wiping. If the slide was located too close to the source the deposited material was granular and had a tendency to flake. For these experiments this distance was about $10 \mathrm{~cm}$, but we expect the distance to increase for larger beam areas on the dump surface. Deposits were found only in areas with a direct line of sight to the target.

From these results, a worst-case prediction of the thickness of deposited material on the spatial filter lenses can be made. We assume that there are no collisions, that the surface normal is at $+60^{\circ}$ to the incoming beam, that the ablated distribution is independent of the beam area, and that all material outside the solid angle of the lens is stopped by the beam dump geometry. For the CSF and TSF, whose focal lengths are 1200 and $3000 \mathrm{~cm}$, respectively, the data in Figure 4 indicate the following:

thickness/shot on the CSF lens: $(1 / 1200)^{2} \times 2 \mathrm{~nm} \times(0.125 / 0.002)=8.7 \times 10^{-5} \mathrm{~nm}$

thickness/shot on the TSF lens: $(1 / 3000)^{2} \times 2 \mathrm{~nm} \times(0.625 / 0.002)=6.9 \times 10^{-5} \mathrm{~nm}$.

The first factor in parentheses assumes an inverse square law dependence, and the second assumes the quantity of ablated material scales directly with the beam area. Therefore a layer of less than $10^{-4} \mathrm{~nm}$ will be deposited per shot at the maximum expected fluence for either lens. Since this is less than a monolayer, it is expected to pose no threat to the optics. The residual vacuum in the spatial filters will be about 0.1 mtorr, which translates into a mean free path of approximately $50 \mathrm{~cm}$. Since an ablated particle will undergo an average of 24 and 60 collisions, respectively, on the way to the CSF lens and TSF lens, we can expect the above thicknesses to be overestimates.

\section{CONCEPTUAL NIF DESIGN}

These measurements, along with observations of the morphology of the deposits on the slides, provided the basis of the design of a suitable beam dump to be used in the NIF spatial filters. The basic design philosophy is to build a collecting box about the intercepting surface which collects all ablated material except that which is emitted with a direct line of sight to the lens, as shown in Figure 5. The intercepting surface normal will be at least $60^{\circ}$ from the beam 


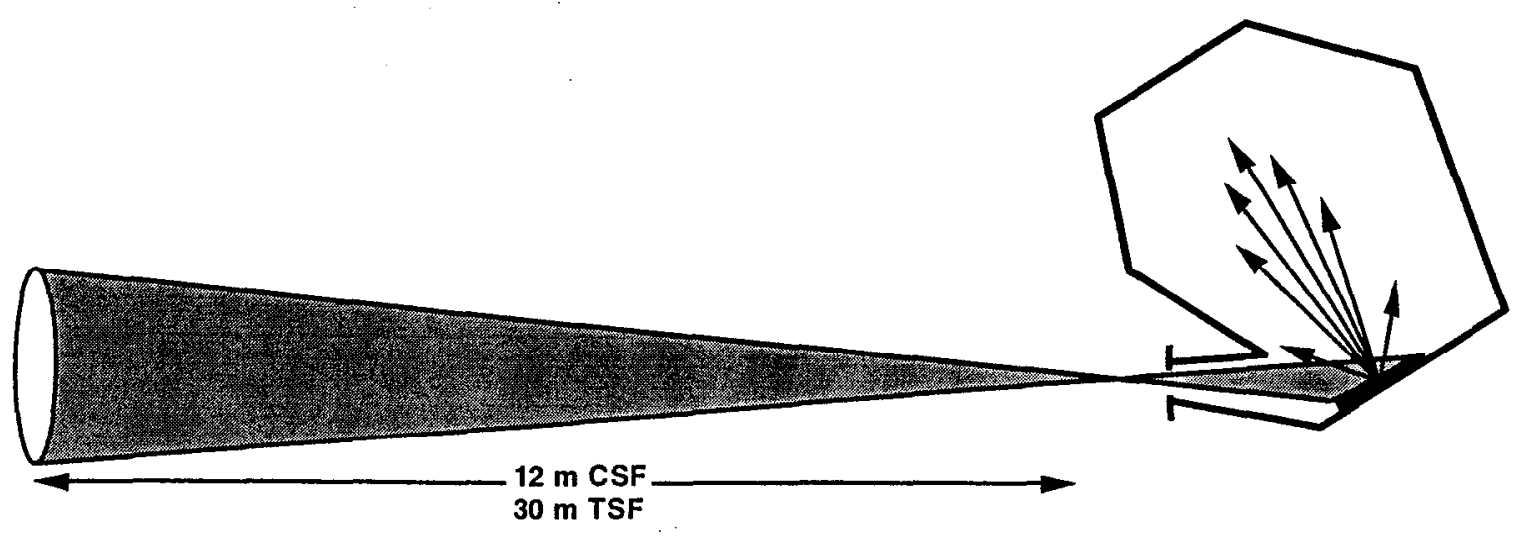

Figure 5. Schematic of beam dump concept.

direction and will be fabricated of stainless steel with a thickness of $1 \mathrm{~cm}$. The rest of the box is also constructed of stainless steel, with 1-mm thick walls. The entrance hole must be sufficiently large to allow easy alignment.

The first collecting surface will be located ten or more centimeters from the intercepting surface to insure that ablated products adhere to the surface and are collected. Since there was no evidence that the ablation products reach beyond the line of sight, it is expected that the containment volume will entrap all the ablation products except those directed back along the beam path. For low-fluence shots, for which the vapor does not absorb the laser pulse, the box geometry will contain the laser pulse with multiple reflections. Escaping laser photons then offer no threat.

\section{CONCLUSIONS}

Off-line experiments have demonstrated the viability of a stainless steel beam dump for use at fluence levels up to $10 \mathrm{~kJ} / \mathrm{cm}^{2}$. The experiments are partially understood on the basis of modeling. Estimates obtained from experimental distributions suggest that there will be inconsequential contamination of the spatial filter lenses. The proposed dump geometry will minimize the contamination of nearby components, remain intact for thousands of shots, and require no maintenance. 


\section{ACKNOWLEDGEMENTS}

*Work performed under the auspices of the U.S. Department of Energy by Lawrence Livermore National Laboratory under Contract No. W-7405-Eng-48.

\section{REFERENCES}

1. C. B. Dane, L. E. Zapata, W. A. Neuman, M. A. Norton, and L. A. Hackel, "Design and Operation of a $150 \mathrm{~W}$ Near Diffraction-Limited Laser Amplifier with SBS Wavefront Correction", IEEE JOE, 31, 148-163 (1995).

2. C. D. Boley and J. T. Early, "Computational Model of Drilling with High Radiance Pulsed Lasers," Proc. Intem. Conf. on Applications of Lasers and Electro-Optics," Orlando, FL, Vol. 79, pp. 499-508 (1994). 